\title{
Las tic en el aprendizaje universitario, según la edad ${ }^{1}$
}

\author{
Ana Fores Miravalle²
}

Fecha de recepción: 13 de junio de 2013

Fecha de revisión: 12 de julio de 2013

Fecha de aprobación: 11 agosto de 2013

\section{Resumen}

Este artículo recoge los resultados finales de la investigación financiada sobre los Usos de las tecnologías de la información y la comunicación (TIC) entre los estudiantes universitarios con relación a sus procesos de aprendizaje. Se ha investigado para conocer mejor cómo tiene lugar la construcción de la identidad de los jóvenes mediante las TIC y sus implicaciones en los procesos de aprendizaje. En este texto nos centramos en la influencia del factor edad en el tipo de uso de las TIC, en la universidad y fuera de ella. La metodología de la investigación ha consistido, en una primera fase, en la aplicación de un cuestionario para recoger la opinión de las y los estudiantes de una muestra de cinco universidades y distintas titulaciones. La intención fue constituir una muestra diversa que permitiera posteriormente construir perfiles con base a distintos criterios, uno de ellos la edad. La muestra final ha sido de 10681 estudiantes.

\section{Palabras clave:}

Tecnologías, universidad, estudiantes, aprendizaje, educación informal.

1 Artículo de investigación financiado que presenta resultados finales fruto del Proyecto $I+D$ : Usos de las TIC entre los estudiantes universitarios: perspectiva académica y social de los procesos de aprendizaje mediados (EDU2009-12125 financiado en España por el Ministerio de Ciencia e Innovación).

2 Doctora en Filosofía y Ciencias de la Educación por la UB, docente de la Facultad de Educación de la Universidad de Barcelona, escritora de numerosos artículos y libros desde el ámbito educativo y directora de proyectos investigativos sobre educación, pedagogía y sociedad. Correo: annaforesub@gmail.com 


\section{Ict in college learning, by age}

\section{Abstract}

This article presents the results of a research on Information and Communication Technology usage among college students, in relation to their learning processes. The aim of this research is to better understand the construction of youngsters' identity through ICT, and its implications in the learning process. In this paper, we focus on the influence of the age factor on the type of use of ICT, in college and beyond. In a first phase, the research methodology consisted in questionnaire filling, to collect the views of students of a sample of five different universities and degrees. The intention was to provide a diverse sample, which would enable building profiles based on different criteria, for instance the age. The final sample has consisted of 10,681 students. To get a more deep insight into some of the questionnaires data, several discussion groups have been created.

\section{Keywords}

Technology, University, Student Learning, Informal Education.

\section{As tic na aprendizagem universitário segundo a idade.}

\section{Resumo}

Este artigo recolhe os resultados finais da pesquisa financiada sobre os Usos da tecnologia da informação e comunicação (TIC) entre os estudantes universitários em relação a seus processos de aprendizagem. Foi-se pesquisado para entender melhor como se têm dado a construção da identidade dos jovens através das TIC e as suas implicações nos processos de aprendizagem. Neste artigo vamos-nos concentrar na influência do fator idade no tipo de utilização das TIC, na universidade e além dela. A metodologia da pesquisa consistiu, numa primeira fase, na aplicação dum questionário para colher as opiniões das e dos alunos de uma amostra de 
cinco universidades e diferentes formaturas. A intenção foi construir uma amostra diversificada que permitisse construir perfis baseados em diferentes critérios, um deles a idade. A amostra final têm sido de 10.681 alunos.

\section{Palavras-Chave:}

Tecnologias, universidade, estudantes, aprendizagem, educação informal.

\section{Introducción}

El proyecto I+D de investigación "Usos de las TIC entre los estudiantes universitarios: perspectiva académica y social de los procesos de aprendizaje mediados por TIC", desarrollado entre 2009 y 2012 ha permitido identificar y comprender mejor el uso de las tecnologías de la información y la comunicación por parte de estudiantes universitarios de universidades de Cataluña, con relación a sus procesos de aprendizaje.

La investigación ha centrado su estudio en el uso de las TIC en situaciones de aprendizaje universitario, contemplando tanto que dicho uso puede ser motivado por parte del profesorado, como que puede ser desarrollado de manera autónoma por el o la estudiante. Se ha investigado respecto de cuáles son las tecnologías utilizadas por los y las estudiantes universitarios, las actividades y acciones que su uso les permiten, y las actitudes y preferencias hacia dichas tecnologías. El campo de estudio de las TIC y su influencia en los procesos de aprendizaje tiene ya una tradición académica consolidada, tal y como se desprende del análisis de la bibliografía que se reseña al final del artículo. Sin embargo, el estudio sistemático centrado en el uso que hacen las y los estudiantes de las TIC y la influencia de dichas prácticas en sus procesos de aprendizaje, solamente ha empezado a ser abordado en los últimos años.

\section{Finalidad e interés de la investigación}

Esta investigación tiene como finalidad conocer el uso de las TIC en los procesos de aprendizaje de los y las estudiantes universitarios, en un sentido transversal e integral. Es decir, con una visión que asume que tanto los usos propuestos en las actividades académicas planteadas en el programa de cada asignatura, como 
otras aplicaciones que los estudiantes realizan por propia iniciativa pueden tener una relación directa o indirecta con las tareas académicas, pero que, en todo caso, esta utilización implica diferentes procesos de aprendizaje. Con el empleo de las TIC se aprende, sea esta inducida o espontánea.

En las situaciones de aprendizaje de las y los estudiantes se dan, en general, actividades y entornos de aprendizaje, y se utilizan herramientas y recursos, teniendo siempre en cuenta el planteamiento y las orientaciones del docente.

La hipótesis de inicio de la investigación consideraba que los y las estudiantes utilizan distintas herramientas tecnológicas y lo hacen de forma diferenciada según si su función se refiera al ámbito cognitivo, al didáctico o al social, y que este uso diferenciado está determinado por las finalidades inherentes a cada medio, por el tipo de acciones y tareas a desarrollar y también por las diferentes variables (edad, tipología de enseñanza, sexo y ámbito académico).

La investigación pretendía explicar hasta qué punto intervienen estos factores, de qué forma lo hacen y cómo interaccionan entre ellos, considerando la posible correlación de los usos de las tecnologías con los resultados obtenidos. Además, consideramos que el uso que los jóvenes hacen de las TIC en los diferentes contextos o ámbitos identificados determina de manera importante sus procesos de aprendizaje en situaciones formales.

Pretendemos que los resultados de esta investigación pueden contribuir, junto con otros, a mejorar las propuestas formativas en el ámbito universitario, a partir de un diseño de las situaciones y de entornos del aprendizaje mediados por las TIC más adaptado al perfil de los y las estudiantes actuales, y ser más coherentes con las formas en que estos construyen sus aprendizajes, ya sea individualmente o en comunidad.

\section{Objetivos de la investigación}

- Analizar los usos de las TIC que realizan los y las estudiantes universitarios, asociados a procesos de aprendizaje.

- Caracterizar el tipo de uso, las preferencias y las actitudes hacia las TIC desarrolladas por parte de los y las estudiantes universitarios en situaciones de aprendizaje.

- Observar la relación entre las TIC utilizadas, los ámbitos de aprendizaje, las acciones/tareas a desarrollar y las diferentes variables (edad, tipología de enseñanza, sexo, ámbito académico). 
- Elaborar un catálogo de buenas prácticas de aprendizaje con TIC útil al profesorado para una mejor comprensión de los procesos de aprendizaje universitario mediados por tecnologías que orienten el diseño de situaciones de enseñanza y aprendizaje en contextos formales.

\section{El estudio de los usos de las TIC según la edad}

De las variables que se han considerado en la investigación, en este artículo nos centramos en la edad de las y los estudiantes. El uso de las TIC con relación a la edad está aún poco estudiado, y especialmente si nos referimos a su relación con el aprendizaje universitario.

En ocasiones, se dice que las y los estudiantes universitarios ya son personas adultas, pero ello no es cierto, como mínimo desde determinados puntos de vista. Si por persona adulta entendemos un concepto que va más allá del ámbito biológico y consideramos también las perspectivas psicológica o de posición social, entre otras, no podemos etiquetar de igual manera a jóvenes de 17 o 18 años -algunos de los cuales aún podríamos considerar en una fase final de la adolescencia- que a personas de 30 o más años. Es evidente que, en la sociedad actual, se suele considerar jóvenes a personas que antaño ya eran conceptualizadas como adultas.

Por otra parte, la rápida y generalizada expansión del fenómeno de las nuevas tecnologías de la información y de la comunicación está suponiendo algo más que un nuevo recurso, para influir en la manera de captar el mundo, de relacionarse o de aprender. Se ha hablado de cambio de paradigma, de nativos y de turistas digitales, y de una brecha digital entre las personas según su edad. Es por todo ello, que nos pareció relevante estudiar el uso de las tecnologías en el contexto que ya hemos señalado, con relación a la edad.

\section{Antecedentes y estado actual del tema}

Los antecedentes del proyecto se encuentran en tres ámbitos temáticos:

- El concepto de aprendizaje a lo largo de la vida como marco de análisis y de comprensión de los procesos de aprendizaje mediados por las TIC, con espacios de intersección entre usos didácticos, cognitivos y sociales, así como académicos y profesionales. 
- El proceso de construcción de la identidad (personal/ social/profesional/ académica) de las y los estudiantes a través del uso de las TIC con relación a los procesos de aprendizaje.

- El papel de las TIC en el logro de competencias académicas / profesionales.

Creemos que la educación superior tiene grandes desafíos a enfrentar, partiendo del hecho de que tanto estudiantes como profesores tendrán una gran responsabilidad en la construcción de las denominadas sociedades del conocimiento. Los jóvenes (estudiantes, en nuestro caso) están llamados a desempeñar un papel fundamental en este ámbito, ya que suelen hallarse a la vanguardia de la utilización de las nuevas tecnologías y contribuyen a insertar la práctica de estas en la vida diaria. Los docentes también están destinados a desempeñar un papel importante, porque cuentan con la experiencia necesaria para compensar la relativa superficialidad de la comunicación "en tiempo real" y recordarnos que "el conocimiento es esencialmente un camino hacía la sabiduría" (Unesco, 2005).

El campo de estudio de las TIC y su influencia en los procesos de aprendizaje se ha visto enriquecido con el trabajo impulsado por la Comisión Europea a través de distintos programas y convocatorias. El estudio sistemático del uso que hacen de las TIC las y los estudiantes, y la influencia en sus procesos de aprendizaje se ha empezado a realizar hace pocos años, como muestran trabajos como los de García Carrasco y Álvarez (2008), González Mariño (2008), Riley (2007) y Veltman (2006).

En nuestro contexto más inmediato, hemos tomado como referencia diversas investigaciones relacionadas con el análisis de procesos de enseñanza y aprendizaje mediados por tecnologías coordinadas o en las que ha participado el grupo de investigación sobre Entornos y Materiales para el Aprendizaje (EMA) de la Universidad de Barcelona: Proyecto Internet Cataluña (PIC), liderado por la Universitat Oberta de Catalunya (UOC), el subproyecto PIC Universidades, dirigido por Josep $\mathrm{M}^{\mathrm{a}}$. Duart, los proyectos Aprendizaje colaborativo y trabajo por proyectos mediante el aula virtual, subvencionado por la Universidad de Barcelona y dirigido por Anna Escofet y la Adopción de las TIC para la construcción colaborativa de conocimiento en el ámbito escolar dirigido por Begoña Gros. Estos proyectos, junto con diversas tesis doctorales, constatan el relieve que creemos que tiene la investigación sobre el rol y la acción del estudiante con relación a sus prácticas mediadas por TIC en el proceso de enseñanza y aprendizaje, y que responden a la necesidad de investigar dichas prácticas para conseguir una mejor comprensión del aprendizaje universitario. 


\section{Metodología de investigación}

Aunque el diseño de investigación del proyecto es más amplio, este artículo se centra en el proceso de recogida y análisis de datos y en los resultados relacionados con el factor edad.

La investigación se proponía ampliar el conocimiento sobre la realidad educativa en el contexto universitario, a partir de las acciones, percepciones y significados atribuidos al uso de las TIC en los procesos de aprendizaje por parte de los propios agentes implicados: los y las estudiantes universitarios. Respondiendo a esta intencionalidad, la metodología de investigación ha consistido, en una primera fase, en la aplicación de un cuestionario para recoger la opinión de las y los estudiantes de una muestra de cinco universidades catalanas y distintas titulaciones. La intención fue constituir una muestra diversa de estudiantes que permitiera, posteriormente construir perfiles con base a distintos criterios, la edad entre ellos.

Así pues, a partir del análisis de los datos recogidos se extrajeron distintos perfiles para un análisis específico de cada perfil. Para profundizar en algunos de los datos obtenidos mediante los cuestionarios, se han realizado grupos de discusión.

\section{Muestra}

La población analizada han sido las y los estudiantes matriculados durante el curso 2010-2011 en los grados de primero a cuarto de cinco universidades catalanas: tres públicas (Universidad de Barcelona, Universidad de Lleida y Universidad Politécnica de Cataluña), una privada (Universidad de VIC) y una de formación a distancia (Universidad Oberta de Catalunya).

La muestra final de estudiantes que han participado en la investigación ha sido de un total de 10681 personas (error: 5\%, margen de confianza: 95,5\%). La selección de la muestra se hizo de manera aleatoria.

\section{Instrumentos y procedimiento}

Para el análisis y la interpretación de los datos se pensó en utilizar una doble aproximación:

- Cuantitativa y estadística mediante el cuestionario y la monitorización de tareas, con el fin de analizar las correlaciones entre las variables analizadas y la 
extracción de patrones de uso referidos a los distintos perfiles de estudiantes. Para los cálculos estadísticos se utilizó el programa SPSS.

- Cualitativa mediante el análisis de contenido de los datos recogidos en los grupos de discusión (se ha utilizado el programa de análisis cualitativo Atlas.ti).

Para complementar la perspectiva de las y los estudiantes, se ha realizado una sesión de trabajo con profesores especializados en el uso de las TIC en la universidad. Con ellos, se han contrastado los datos obtenidos de las y los estudiantes y se ha reflexionado sobre cómo potenciar buenas prácticas en el uso de las TIC.

Como variables independientes en la investigación se han considerado las siguientes:
a) Edad,
b) Sexo,
c) Ocupación,
d) Capacitación y equipamiento tecnológico,
e) Alcance del conocimiento,
f) Tipo de enseñanza (virtual /mixta o blended),
g) Tipo de universidad,
h) Nivel académico / curso.

Se han explotado a fondo los datos correspondientes a cuatro de estas variables, entre ellas la edad.

Los datos recogidos por el cuestionario se refieren a los siguientes aspectos:

1. Tipos de usos de las TIC relacionados con las tareas de aprendizaje académico (tanto espontáneo como bajo petición del profesorado).

2. Las actitudes y preferencias de los estudiantes hacia ciertas tecnologías y prácticas en la universidad (utilidad percibida, la actitud hacia el uso de las TIC).

3. Aspectos cognoscitivos, sociales y didácticos del uso de las TIC en la universidad.

4. Los usos informales de las TIC (frecuencia y nivel de competencia) y definición de perfiles de uso.

5. La utilización informal de las TIC frente a las aplicaciones relacionadas con los procesos de aprendizaje (espontáneos y dirigidos): las intersecciones y contradicciones. 
Los grupos de discusión formados con estudiantes seleccionados que representaban los perfiles identificados, a través del cuestionario han permitido una interpretación más precisa de los resultados, y también un análisis detallado de las prácticas de aprendizaje mediado por ciertos usos de la tecnología y su relación con las tareas académicas.

En este artículo realizamos una explotación de la variable edad. Más allá del análisis descriptivo, se pretende extraer los diferentes perfiles de uso de la tecnología según las edades.

\section{Resultados: uso de las TIC según edad}

A continuación, presentamos los resultados según las franjas de edad que se han contemplado en la investigación: 17-18, 19, 20-21, 22, 23-29, 30 y más años. En primer lugar se señalan las características más destacadas relacionadas con el perfil de cada una de estos márgenes de edad. Seguidamente, se relacionan las edades con el uso formal de las TIC a propuesta del profesorado, la valoración de la utilidad para la propia formación en el uso de las TIC a propuesta de los docentes, el uso formal de las TIC por propia iniciativa, la frecuencia de uso informal de TIC por iniciativa propia, y la competencia en el uso informal de las TIC.

Para cada una de las dimensiones que se han analizado, se presenta una tabla donde se recogen las principales características para cada zona de edad. Se han reunido aquellas respuestas que han aparecido de forma mayoritaria en cada franja. En ocasiones, se ha dado una gran disparidad en las respuestas mientras que en otros casos han primado las coincidencias. Ello explica que en las siguientes tablas, en ocasiones aparezcan pocas respuestas, y en otras estas sean numerosas.

\section{Perfil de cada edad: características más destacadas}

Para cada rango de edad, se señalan las características más sobresalientes encontradas en la muestra seleccionada. Se recoge el perfil universitario (tipo de universidad: presencial o no presencial; curso donde están matriculados; y si compaginan o no el estudio con el trabajo) y las respuestas de los estudiantes de la muestra respecto desde dónde se conectan a Internet. En el diseño de la investigación se consideró que estos datos eran relevantes para caracterizar cada franja de edad con relación al tema objeto de investigación. 
Tabla I. Perfil: características más destacadas

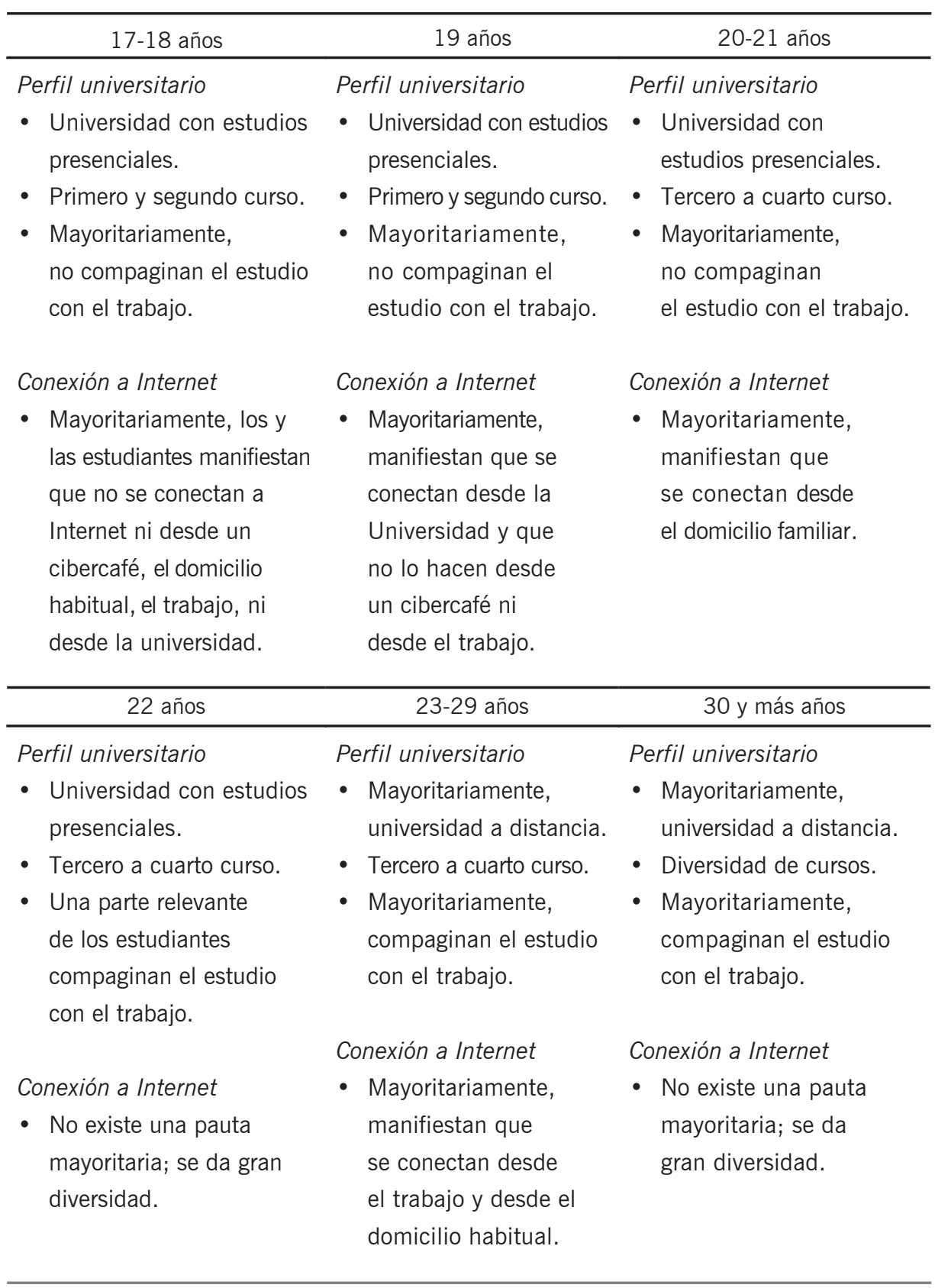


Con relación al perfil, se destaca que a partir del rango de 23-29 años se compaginan estudios y trabajo y predominan los estudios en una universidad con estudios virtuales.

En lo que se refiere a la conexión a Internet, en la franja 17-18 años, se responde que no se conectan a Internet ni desde un cibercafé, ni desde el domicilio habitual, ni desde el trabajo, ni desde la Universidad, lo cual sorprende puesto que indica que no se conectan desde un espacio estable, institucionalizado, por lo que se infiere que se conectan en espacios de medio abierto (la calle, por ejemplo).

A los 19 años aparece la conexión mayoritaria desde la Universidad y en la franja 20-21 años desde el domicilio familiar. En el rango de 23-29 años, donde se compaginan estudios y trabajo y predominan los estudios no presenciales aparece la conexión desde el trabajo y el domicilio habitual. En la siguiente franja de edad, a partir de los 30 años, se da una gran diversidad que puede responder a la variedad de población considerada en un rango tan amplio.

\section{Uso formal de las TIC a propuesta del profesorado}

En esta dimensión se ha estudiado la frecuencia del uso por parte de los y las estudiantes de las TIC cuando este se da a raíz de la propuesta o de las orientaciones del docente. Se trata, por lo tanto, de una utilización de las TIC inducida. En la siguiente tabla se señalan las características que aparecen como más destacadas.

Tabla II. Uso formal de las TIC a propuesta del profesorado

17-18 años

19 años

20-21 años

- Nunca utilizan - Uso frecuente del campus virtual. - Uso frecuente un fórum.

- En alguna ocasión: de Youtube.

- Utilizan repositorios.

- Realizan búsquedas por Internet.

- Emplean la pizarra digital.

- Nunca usan:

- Un fórum

- Wikis. 
- Uso frecuente de la pizarra digital.

- En alguna ocasión:

- Utilizan juegos y simulaciones.

- Usan la webquest.
- Siempre utilizan el Google Docs.

- Uso frecuente de:

- Fórum.

- Wiki.

- En alguna ocasión usan el chat.

(1)


A los 19 años, por ejemplo, se utilizan en alguna ocasión las búsquedas en Internet que son habituales a partir de los 30 años. Los fórum no se utilizan hasta los 19 años, son frecuentes en el rango de 23-29 años y habituales a partir de los 30. A los 19 años se utiliza el campus y en ocasiones repositorios y la pizarra digital, que se usa frecuentemente a los 22 años.

En el margen de 23-29 años se usan frecuentemente wiquis y alguna vez chats. A partir de los 30 años, se emplean numerosos recursos que no aparecen en edades más tempranas o lo hacen sólo en cierta edad (Youtube a los 20-21 años, por ejemplo). Aun así, existen muchas aplicaciones que nunca se utilizan (a propuesta del profesorado) a partir de los 30 años. En esta última franja de edad se destaca tanto el uso mayoritario de determinados recursos como el que en alta proporción no se usan otros recursos (siempre a propuesta del docente), a diferencia de otros rangos de edad en los cuales existe más diversidad entre el alumnado (en estas otras franjas no se da ni un uso ni un no uso mayoritario de tantos recursos).

\section{Valoración de la utilidad para la formación de las TIC utilizadas a propuesta del profesorado}

Complementando la dimensión anterior, a continuación se recoge la percepción del alumnado sobre el grado de utilidad del uso de las TIC a propuesta del profesorado. Se trata de ver hasta qué punto se considera que este uso mejora la propia formación. En la siguiente tabla, se han recogido las respuestas del alumnado a dos apartados del cuestionario: grados de utilidad de los diversos recursos y de acuerdo con una serie de afirmaciones referidas a la utilidad de las TIC, en general.

Tabla III. Valoración de la utilidad para la formación de las TIC utilizadas a propuesta del profesorado

\begin{tabular}{ll}
\hline 17-18 años & \multicolumn{1}{c}{ 20-21 años } \\
\hline & \\
& - Muy útil: wiki \\
& - Búsquedas en Internet. \\
& - Adquisición de conocimientos \\
& relacionados con la asignatura. \\
\hline
\end{tabular}


- Las TIC no son útiles para:

- Mejorar la comunicación con el profesor o profesora

- Que el profesorado paute la metodología de trabajo.

- Hacer preguntas a los compañeros y compañeras.

- Diagnosticar los propios errores de aprendizaje.

\begin{tabular}{|c|c|c|}
\hline 22 años & 23-29 años & ños \\
\hline $\begin{array}{l}\text { - Muy útil: el chat. } \\
\text { - Útil: } \\
\text { - Repositorios } \\
\text { de información. } \\
\text { - Fórum. } \\
\text { - Juegos y simulacio- } \\
\text { nes (para una } \\
\text { parte relevante } \\
\text { de la muestra: } \\
\text { son muy útiles). } \\
\text { - Las TIC son útiles } \\
\text { para potenciar el } \\
\text { ritmo de trabajo } \\
\text { y para facilitar } \\
\text { la presentación } \\
\text { de contenidos. } \\
\text { Para una parte relevante } \\
\text { de la muestra el } \\
\text { Webquest es útil } \\
\text { mientras que para } \\
\text { otra, también } \\
\text { relevante, no lo es. }\end{array}$ & $\begin{array}{l}\text { - Muy útil/ muy } \\
\text { de acuerdo en } \\
\text { la utilidad: } \\
\text { - Repositorios } \\
\text { de información. } \\
\text { - Webquest. } \\
\text { - Goog/e Docs. } \\
\text { - Las TIC } \\
\text { permiten } \\
\text { una mejor } \\
\text { comunicación } \\
\text { con } \\
\text { el profesor } \\
\text { o profesora. } \\
\text { - Útil: } \\
\text { - Podcast } \\
\text { - Video/audio } \\
\text { clip. } \\
\text { - Foutube. } \\
\text { Fotografía } \\
\text { digital. }\end{array}$ & $\begin{array}{l}\text { - Muy de acuerdo con que las TIC: } \\
\text { - Permiten planificar el trabajo } \\
\text { - Ael alumnado. } \\
\text { y habilidades relacionadas con } \\
\text { la asignatura. } \\
\text { - Permiten valorar mejor el progreso } \\
\text { en la asignatura. } \\
\text { - Posibilita aplicar el conocimiento } \\
\text { adquirido. } \\
\text { - Potencian el ritmo de trabajo. } \\
\text { - Hacen más fácil aprobar la asignatura. } \\
\text { - Permiten intercambiar ideas con } \\
\text { el profesorado. } \\
\text { - Facilitan al profesorado pautar } \\
\text { la metodología. } \\
\text { - Facilitan la integración de conocimientos } \\
\text { - de distintas fuentes. } \\
\text { - Eacilitan el establecimiento de un } \\
\text { clima agradable. } \\
\text { a realizar mejor las tareas universitarias. }\end{array}$ \\
\hline
\end{tabular}


La utilidad de las TIC en la formación a propuesta del profesorado se percibe de manera distinta según las edades. A los 20-21 años se consideran muy útiles las wikis y útiles la búsqueda en Internet y compartir conocimientos y, en cambio no se considera útil el Chat ni se cree que las TIC faciliten aspectos como la comunicación con el profesorado, hacer preguntas a los demás o diagnosticar los errores de aprendizaje, entre otros aspectos.

A los 22 años se considera muy útil el Chat y útiles los repositorios, los fórum y los juegos y simulaciones; en esta edad se considera que las TIC tienen diversas utilidades.

A los 23-29 años, se consideran muy útiles los repositorios, el Goog/e Docs y el Webquest (que a los 22 años tenía partidarios y detractores) y útiles el Podcast, el vídeo/audio clip, el Youtube y la fotografía digital. En esta franja de edad se considera que las TIC permiten una mejor comunicación con el profesorado.

A partir de los 30 años, se valora la utilidad de las TIC para planificar el trabajo, valorar el progreso de la asignatura, aplicar el conocimiento adquirido, potenciar el ritmo de trabajo y otros aspectos, aunque sorprende que se manifiesta no estar de acuerdo con que las TIC ayuden a hacer mejor las tareas universitarias.

\section{Uso formal de las TIC por iniciativa propia}

Esta dimensión explora el uso de las TIC por iniciativa propia cuando este uso se hace para las tareas académicas. Hemos resaltado los recursos que el alumnado manifiesta utilizar mayoritariamente.

Tabla IV. Uso formal de las TIC por iniciativa propia

17-18 años

19 años

20-21 años

- Redes sociales.

- Intercambio

de fotografías

por Internet.

- Internet para jugar

(en menor medida). 


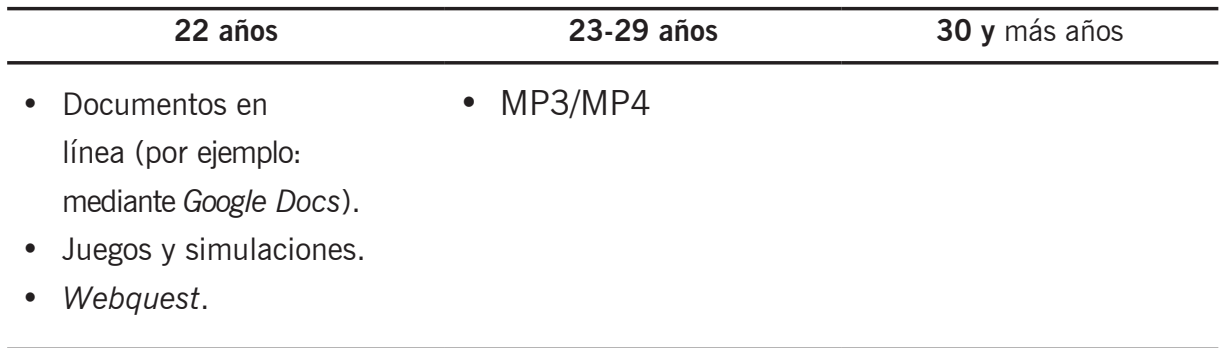

El uso formal de las TIC (para tareas académicas) por propia iniciativa, a los 17-18 años parece inexistente, a los 19 años se utilizan redes sociales y se usa Internet para jugar (en alguna ocasión) y frecuentemente para intercambiar fotografías.

A los 22 años se trabaja con documentos en línea, con juegos y simulaciones y webquest.

En la franja 23-29 años se usa el MP3/MP4.

El uso significativo por iniciativa propia parece desaparecer a partir de los 30 años.

\section{Uso informal de las TIC por iniciativa propia: frecuencia de uso}

Esta dimensión recoge la frecuencia en el uso informal de las TIC (no dirigido a la utilidad académica).

Tabla V. Uso informal de las TIC por iniciativa propia: frecuencia de uso

\begin{tabular}{ccc}
\hline 17-18 años & \multicolumn{1}{c}{ 19 años } & \multicolumn{1}{c}{ 20-21 años } \\
\hline - Uso diario: & - Uso diario: & - Alguna vez a la semana: \\
- Internet para & - Internet para & - Internet para hacer \\
mantener & mantener comunicación & videoconferencias. \\
comunicación con & con amigos o amigas. & - Internet para buscar \\
amigos o amigas. & - Internet para participar & información general. \\
- Internet para & en una red social. & - Alguna vez al mes: \\
chatear. & - Ordenador para & - Internet para \\
- Internet para & escuchar música. & crear y mantener \\
participar en & - Teléfono móvil & el propio blog. \\
una red social. & para MP3. & \\
& & \\
\hline
\end{tabular}




\begin{tabular}{|c|c|c|}
\hline 17-18 años & 19 años & 20-21 años \\
\hline $\begin{array}{l}\text { - Internet y el } \\
\text { ordenador para } \\
\text { escuchar música. } \\
\text { - Nunca se usa: } \\
\text { - Internet para } \\
\text { comprar o vender. } \\
\text { - Internet para } \\
\text { videoconferencias. }\end{array}$ & $\begin{array}{l}\text { - Teléfono móvil para } \\
\text { chatear.Teléfono móvil } \\
\text { para leer blogs. }\end{array}$ & $\begin{array}{l}\text { - Teléfono móvil } \\
\text { para hacer } \\
\text { videollamadas. }\end{array}$ \\
\hline 22 años & 23-29 años & 30 y más años \\
\hline $\begin{array}{l}\text { - Uso diario: } \\
\text { - Ordenador para } \\
\text { jugar (también para } \\
\text { jugar en red). } \\
\text { - Teléfono móvil para } \\
\text { acceder a Internet. } \\
\text { - Ordenador para } \\
\text { crear o manipular } \\
\text { imágenes. } \\
\text { - Internet para bajar } \\
\text { y publicar podcast. } \\
\text { - Internet para com- } \\
\text { prar o vender. } \\
\text { - Alguna vez al mes: } \\
\text { - Teléfono móvil para } \\
\text { enviar fotografías o } \\
\text { vídeos. } \\
\text { - Internet para con- } \\
\text { tribuir al desarrollo } \\
\text { social. }\end{array}$ & $\begin{array}{l}\text { - Uso diario: } \\
\text { - Ordenador para jugar } \\
\text { - Ordenador para } \\
\text { crear o manipular } \\
\text { imágenes. } \\
\text { - Internet para descargar } \\
\text { software y películas. } \\
\text { - Internet para enviar } \\
\text { sms. } \\
\text { - Internet para } \\
\text { acceder a medios } \\
\text { de comunicación. } \\
\text { - Internet para } \\
\text { traducir textos. } \\
\text { - Internet para recibir } \\
\text { y enviar correo. } \\
\text { - Marcadores sociales. } \\
\text { - Teléfono móvil para } \\
\text { acceder a Internet. } \\
\text { - Teléfono móvil } \\
\text { para hacer fotografías } \\
\text { o vídeos. }\end{array}$ & $\begin{array}{l}\text { - Uso diario: } \\
\text { - Internet para acceder } \\
\text { al campus virtual. } \\
\text { - Internet para reci- } \\
\text { bir y enviar correo } \\
\text { electrónico. } \\
\text { - Internet para buscar } \\
\text { información general. } \\
\text { - Alguna vez a la } \\
\text { semana: } \\
\text { - Internet para mantener } \\
\text { comunicación con } \\
\text { amigos o amigas. } \\
\text { - Alguna vez al mes: } \\
\text { - Ordenador para } \\
\text { escuchar música. } \\
\text { - Internet para comprar } \\
\text { y vender. } \\
\text { - Internet para escuchar } \\
\text { música. } \\
\text { - Internet para mantener } \\
\text { comunicación con } \\
\text { amigos o amigas. }\end{array}$ \\
\hline
\end{tabular}




\begin{tabular}{|c|c|c|}
\hline 22 años & 23-29 años & 30 y más años \\
\hline & $\begin{array}{l}\text { - Alguna vez a la semana: } \\
\text { - Ordenador para crear } \\
\text { o editar audio y vídeo. } \\
\text { - Internet para leer } \\
\text { blogs y/o fotoblogs. } \\
\text { - Alguna vez al mes: } \\
\text { - Internet para mantener } \\
\text { comunicación con } \\
\text { amigos o amigas. } \\
\text { - Internet para participar } \\
\text { en una red social. } \\
\text { - Internet para hacer } \\
\text { videoconferencias } \\
\text { - Nunca se usa Internet } \\
\text { para gestionar } \\
\text { información online. }\end{array}$ & $\begin{array}{l}\text { - Internet para participar } \\
\text { en una red social. } \\
\text { - Internet para } \\
\text { descargar software } \\
\text { y películas. } \\
\text { - Internet para chatear. } \\
\text { - Internet para compartir } \\
\text { fotografías. } \\
\text { - Teléfono móvil para } \\
\text { hacer fotografías } \\
\text { o vídeos. }\end{array}$ \\
\hline
\end{tabular}

En lo que se refiere al uso informal de las TIC en la franja 17-18 años se usa Internet diariamente para relacionarse (comunicarse con algún amigo, chatear, participar en una red social) y para escuchar música. También se usa diariamente el ordenador para escuchar música.

A los 19 años, a los usos anteriores diarios se añade la lectura de blogs y el uso del teléfono móvil para MP3 y para chatear.

A los 20-21 años aparece el uso de Internet alguna vez a la semana para hacer videoconferencias y para buscar información general, y alguna vez al mes para crear y mantener un blog. También se utiliza el teléfono móvil para vídeollamadas.

A los 22 años, el uso diario es para jugar (también en red). Una vez a la semana para bajar y publicar podcast, para comprar o vender y para crear o manipular imágenes (mediante el ordenador). Alguna vez al mes, se usa el teléfono móvil para enviar fotografías o vídeos e Internet para contribuir al desarrollo.

En la franja 23-29 años, el uso diario es muy variado (jugar, descargar software, enviar sms, correo, traducir textos, etc.). Con un uso menor, se utiliza para crear o editar vídeo, leer blogs, participar en una red social, videoconferencias, etc. El uso es parecido a partir de los 30 años, aunque con una menor intensidad de los aspectos más lúdicos. 


\section{Competencia en el uso informal de las TIC}

Completando el ámbito anterior que se refería al uso informal de las TIC, en este apartado se recoge la percepción que tienen los y las estudiantes sobre su grado de competencia en su utilización.

Tabla VI. Competencia en el uso informal de las TIC

\begin{tabular}{|c|c|c|}
\hline 17-18 años & 19 años & 20-21 años \\
\hline $\begin{array}{l}\text { - Competencia alta } \\
\text { en usar: } \\
\text { - Internet para } \\
\text { mantener } \\
\text { comunicación. } \\
\text { - Internet para } \\
\text { participar en una red. } \\
\text { - Competencia baja } \\
\text { en usar Internet para } \\
\text { traducir textos. } \\
\text { - Ninguna competencia } \\
\text { en usar el teléfono } \\
\text { móvil para enviar } \\
\text { y recibir correos. }\end{array}$ & $\begin{array}{l}\text { - Competencia alta } \\
\text { en crear y mantener } \\
\text { una página web. }\end{array}$ & $\begin{array}{l}\text { - Competencia mediana } \\
\text { en usar: } \\
\text { - Internet para descargar } \\
\text { software. } \\
\text { - Internet para comprar } \\
\text { y vender. } \\
\text { - Internet para participar } \\
\text { en una red. } \\
\text { - ordenador para crear } \\
\text { imágenes. } \\
\text { - Ninguna competencia } \\
\text { en usar: } \\
\text { - Teléfono móvil para } \\
\text { enviar fotografías } \\
\text { o vídeos. } \\
\text { - Teléfono móvil para } \\
\text { enviar y recibir correos. }\end{array}$ \\
\hline 22 años & 23-29 años & 30 y más años \\
\hline $\begin{array}{l}\text { - Competencia alta } \\
\text { en usar: } \\
\text { - Internet para } \\
\text { compartir MP3. } \\
\text { - Internet para } \\
\text { descargar software. }\end{array}$ & $\begin{array}{l}\text { - Competencia alta } \\
\text { en usar: } \\
\text { - Internet para } \\
\text { compartir MP3. } \\
\text { - Internet para } \\
\text { descargar software. }\end{array}$ & $\begin{array}{l}\text { - Competencia alta en usar: } \\
\text { - Internet para buscar } \\
\text { información. } \\
\text { - Internet para acceder } \\
\text { al campus. }\end{array}$ \\
\hline
\end{tabular}




\section{2 años}

- Competencia baja en usar Internet para realizar Ilamadas telefónicas.
- Internet para traducir textos.

- Internet para compartir fotografías.

- Internet para enviar sms.

- Internet para gestionar información online.

- Internet para realizar llamadas telefónicas.

- Internet para comprar y vender.

- Internet para buscar información.

- Ordenador para escuchar música. Ordenador para jugar.

- Teléfono móvil para enviar fotografías o vídeos.

- Teléfono móvil para jugar.

- Teléfono móvil para acceder a Internet.

- Teléfono móvil para enviar y recibir correos.

- Teléfono móvil para hacer vídeo.

- Teléfono móvil para hacer fotografías.
- Internet para comprar $y$ vender.

- Internet para recibir y enviar correos.

- Competencia mediana en usar el ordenador para escuchar música.

- Competencia baja en:

- Descargar software.

- Usar Internet para mantener comunicación.

- Usar Internet para bajar y publicar podcast.

- Usar Internet para leer contenidos.

- Chatear.

- Usar el ordenador para escuchar música.

- Ninguna competencia en:

- Participar en una red social.

- Usar el ordenador para jugar (también en red).

- Usar Internet para crear y mantener una página web.

- Usar el teléfono móvil para jugar. 
En lo que se refiere a las competencias en el uso informal de las TIC, a los 17-18 años se manifiesta una alta competencia en el uso de Internet para comunicarse, incluyendo la participación en una red. La competencia es baja en la traducción de textos y se manifiesta falta de competencia en el uso del teléfono móvil para enviar y recibir correos.

A los 19 años se manifiesta alta competencia para crear y mantener una página web.

A los 20-21 años destaca un nivel competencial medio (descargar software, comprar y vender, participar en una red, etc.). Se manifiesta falta de competencia en usar el teléfono móvil para enviar fotos o vídeos y correos.

A los 22 años se manifiesta una competencia baja en usar Internet para realizar Ilamadas telefónicas.

En la franja 23-29 años, la competencia es alta en numerosas actividades: compartir MP3, descargar software, enviar fotos o vídeos con el teléfono móvil, traducir textos y muchas más.

A partir de los 30 años, se manifiesta una competencia alta para buscar información, acceder al campus, comprar y vender y usar el correo, y una competencia mediana en usar el ordenador para escuchar música. Por el contrario, se manifiesta competencia baja para descargar software, chatear, leer contenidos y otras aplicaciones, así como falta de competencia para participar en una red social, crear y mantener una página web y lo que se refiere a usar las TIC para jugar.

\section{Conclusiones}

Del estudio se concluye que en los estudiantes más jóvenes el uso se centra prioritariamente en aspectos lúdicos y en la comunicación y las redes sociales. Esta utilización evoluciona con la edad, haciéndose más diversificado. Las conclusiones cuestionan la idea del "nativo digital": Ios estudiantes más jóvenes probablemente dominan usos restringidos de las TIC, mientras que los estudiantes de más edad saben menos, pero de un espectro de usos más amplio.

Así mismo, se concluye que el perfil del alumnado según su edad muestra que, a partir del rango 23-29 años, mayoritariamente se compaginan los estudios con el trabajo y que, también a partir de esta edad, predominan en la muestra los estudiantes que cursan sus estudios en una universidad no presencial. 
Estos datos no pueden sorprender. En cambio, quizá si resulte sorprendente que, en lo que se refiere a la conexión a Internet, en la franja 17-18 años se responda mayoritariamente que no se conectan a Internet desde ningún espacio estable habitual. Una hipótesis no comprobada es que se conecten con el teléfono móvil. En cambio, a los 19 años ya aparece una conexión predominante desde la universidad y en la siguiente franja de edad (20-21 años) desde el domicilio familiar, y en la siguiente (23-29 años) también desde el trabajo. Es decir, se da una evolución lógica, pero llama la atención la no conexión de la franja de edad más joven.

En la investigación se ha estudiado el uso de las TIC a propuesta del profesorado y la utilización por iniciativa del propio estudiante. Cuando se realiza a indicación de los y las docentes, se observa una evolución que es coherente con lo observado al tratar del perfil: a medida que aumenta la edad, se incrementa el uso de recursos a propuesta del profesorado: por ejemplo, las búsquedas en Internet no son habituales hasta la franja de más edad (30 años o más), el uso del fórum no aparece hasta los 19 años y se va haciendo más habitual a menudo que se avanza en la edad.

En la misma línea, el uso de las TIC para tareas académicas, pero a iniciativa del propio alumnado es prácticamente inexistente en la franja 17-18 años, mientras que en las otras franjas aparecen algunos recursos, hasta la última franja de la muestra (30 años y más) donde se ha visto que se utilizan diversos recursos por indicación del docente pero, por el contrario, casi no se da un uso para tareas académicas por iniciativa del estudiante.

En la valoración de la utilidad de las TIC para la propia formación cuando se usan a propuesta del profesorado, existen diferencias entre las edades respecto de los recursos que se consideran más útiles. Es de destacar que, a medida que se incrementa la edad, se reconocen más utilidades a las TIC para la propia formación y para la comunicación con el profesorado, aunque en la franja de 30 años y más aparecen voces críticas con la utilidad de las TIC para mejorar las tareas universitarias (en general, sin concretar).

El uso de las TIC con finalidades académicas se ha complementado en la investigación con el estudio de su uso informal, sin estas finalidades. Lo más relevante es que se observa una tendencia a que, a medida que se incrementa la edad, se diversifican los recursos que se utilizan. En la franja 17-18 años se manifiesta un uso diario de Internet (aunque en el perfil apareció que sin un espacio estable habitual para la conexión), pero muy focalizado en la comunicación con los demás y en escuchar música. 
Según se va ascendiendo en la edad, van apareciendo nuevos recursos y funciones, aunque la comunicación con amistades y mediante la red social sigue teniendo un lugar importante. A partir de la franja 23-29 años el uso es mucho más variado y prosigue así en la de 30 y más años, aunque en este caso con una menor presencia de los usos más lúdicos.

El estudio del uso informal de las TIC se ha complementado con el estudio de la percepción de los y las estudiantes sobre sus competencias en este uso. Se observa una relación directa entre el uso que se manifiesta y las competencias que se creen tener. Los y las estudiantes de la franja más joven (17-18 años) que utilizan Internet fundamentalmente para comunicarse, tal como se ha comentado, manifiestan una alta competencia en este uso, mientras que se consideran poco competentes en otros. A los 19 años aparece un mayor interés por las páginas web, con la consiguiente competencia. En el siguiente rango de edad (20-21 años), donde aparecen nuevos usos de las TIC, se manifiesta un nivel competencial medio en el uso de diversos recursos, aunque menor en el caso del teléfono móvil. La de 22 años muestra respuestas muy diversificadas.

En la siguiente, la de 23-29 años, se manifiesta una alta competencia en actividades diversas que va en paralelo al uso diversificado de recursos. A partir de los 30 años se incrementan las competencias en actividades relacionadas prioritariamente con un uso como recurso práctico de las TIC (buscar información, acceder al campus, comprar y vender, etc.) mientras que, por el contrario, se manifiesta una baja competencia en aquellos usos en los que se consideran más competentes las franjas de edad más joven (chatear, participar en una red social, usar las TIC para jugar, etc.).

Se observa, pues, una evolución en el uso de las TIC, desde un uso restringido en cuanto a la diversidad y centrado principalmente en el ámbito comunicativo en las edades más jóvenes del estudio, hasta un uso cada vez más diversificado y, a medida que se incrementa la edad, menos lúdico y menos centrado en la comunicación y las redes sociales.

Estas conclusiones ponen en cuestión -al menos parcialmente- la idea del "nativo digital" como una persona que se mueve con soltura en las nuevas tecnologías. Lo que hemos visto es que esta soltura sólo se da en los usos más relacionados con la comunicación y parcialmente en usos lúdicos. Es a medida que encontramos población de más edad que se da un uso más diversificado de las tecnologías y una mayor competencia en usos diversos de ella. Ello explicaría las sorpresas de algún profesor cuando sus jóvenes estudiantes manifiestan más dificultades de las que había previsto para moverse con soltura en usos diversos de las TIC. 
Si recurrimos al tópico que dice que se puede saber mucho de poco o poco de mucho, los estudiantes más jóvenes probablemente sapan mucho de lo que se relaciona con sus usos restringidos de las TIC, mientras que los y las estudiantes de más edad saben menos, pero de un espectro más amplio de usos.

Esta evolución no se contradice en ninguna de las dimensiones estudiadas, incluida la del uso a instancias del docente. Aunque se trata de un estudio limitado y se requieren más investigaciones, se apuntan cuestiones que, como mínimo, matizan algunas afirmaciones que suelen ser habituales sobre la utilización de las TIC, las competencias, los jóvenes y los adultos. Además de la edad, en la investigación también se han analizado otros indicadores, como por ejemplo el género, cuyas conclusiones ayudarán a configurar una idea más amplia de lo que puede estar sucediendo en el uso de las TIC entre la población universitaria.

\section{Referencias Bibliográficas}

Caruso, J. B.; Kvavik, R. (2005). ECAR study of students and information technology 2005: Convenience, connection, control, and learning. EDUCAUSE. Recuperado de http://connect.educause.edu/Library/ECAR/ECARStudyofStudentsandInf/41159

Duart, J. M.; Gil, M.; Pujol, M.; Castaño, J. (2008). La universidad en la sociedad red. Usos de Internet en Educación Superior. Barcelona: Ariel.

García Carrasco, J.; Álvarez; G. (2008). "Reconfiguración como sujetos de comunicación: implicaciones para los ambientes virtuales con fines educativos". En: "Comunicación y construcción del conocimiento en el nuevo espacio tecnológico" [monográfico en línea]. Revista de Universidad y Sociedad del Conocimiento (RUSC). Vol. 5, n. ${ }^{\circ}$ 2. UOC. Recuperado de http://www.uoc.edu/rusc/5/2/dt/esp/conocimiento_tecnologico.html

González Mariño, J.C. (2008). "TIC y la transformación de la práctica educativa en el contexto de las sociedades del conocimiento" [artículo en línea]. En: Revista de Universidad y Sociedad del Conocimiento (RUSC). Vol. 5, N. ${ }^{\circ}$ 2. UOC. Recuperado de http:// www.uoc.edu/rusc/5/2/dt/esp/gonzalez.pdf

Jones, S.; Madden, M. (2002). The Internet goes to college: How students are living in the future with today's technology. Washington DC: Pew Internet / American Life Project. Recuperado de http://www. pewinternet.org/pdfs/PIP_College_Report.pdf

Kennedy, G.; Krause, K.L.; Gray, K.; Judd, T.; Bennett, S.; Maton, K.; Dalgarno, B.; Bishop, A. (2006). "Questioning the Net Generation: A collaborative project in Australian higher 
education". En: Markauskaite, L.; Goodyear, P.; Reimann, P. (Eds.), Who's learning? Whose technology? Proceedings of the 23rd Annual Conference of the Australiasian Society for Computers in Learningin Tertiary Education. Sydney: Sydney University Press, pp. 413-417. Recuperado de http://www.ascilite.org.au/conferences/sydney06/ proceeding/pdf_papers/p160.pdf.

Kennedy, G.E.; Judd, T.S.; Churchward, A.; Gray, K. y Krause, K.L (2008). First year students'experiences with technology: Are they really digital natives? Australasian Journal of EducationalTechnology, 24(1), pp. 108-122

Kirkwood, A.; Price, L. (2005). "Learners and learning in the 21 st century: What do we know aboutstudents' attitudes and experiences of ICT that will help us design courses?". En: Studies in HigherEducation, 30 (3), pp. 257-274.

Prensky, M. (2001). "Digital Natives, digital inmigrants". En: On the Horizon, 9 (5). Recuperado de http://www.marcprensky.com/writing/Prensky\%20-\%20Digital\%20Natives,\%20 Digital\%20Immigrants\%20-\%20Part1.pdf.

Riley, D (2007). "Educational Technology and Practice: Types and Timescales of Change". En: Educational Technology and Society. Vol. 1, N. ${ }^{\circ}$ 10, pp. :85-93.

Trinder, K; Guiller, J; Margaryan, A.; Littlejohn, A; Nicol, D. (2008). "Learning from digitalnatives: bridging formal and informal learning". Research project report. Glasgow: The Higher EducationAcademy. Glasgow Caledonian University. Recuperado de

http://www.academy.gcal.ac.uk/ldn/LDNFinalReport.pdf.

UCL-CIBER Group (2008). Information Behaviour of the Researcher of the Future ('GoogleGeneration' project). London: University College London CIBER Group. British Library and JISC. Recuperado de

http://www.ucl.ac.uk/infostudies/research/ciber/downloads/

UNESCO (2005). Hacia las sociedades del conocimiento. Informe Mundial de la Educación. París: Unesco.

Veltman, K. (2006). Understanding New Media: Augmented Knowledge and Culture. Calgary: Calgary University Press. 
